\title{
Comparative Analyses of Homocitrate Synthase Genes of Ascomycetous Yeasts
}

\author{
Hiromi Nishida \\ Agricultural Bioinformatics Research Unit, Graduate School of Agricultural and Life Sciences, University of Tokyo, \\ Tokyo 113-8657, Japan \\ Correspondence should be addressed to Hiromi Nishida, hnishida@iu.a.u-tokyo.ac.jp
}

Received 8 December 2011; Accepted 12 January 2012

Academic Editor: Shinji Kondo

Copyright ( $\odot 2012$ Hiromi Nishida. This is an open access article distributed under the Creative Commons Attribution License, which permits unrestricted use, distribution, and reproduction in any medium, provided the original work is properly cited.

Most ascomycetous yeasts have 2 homocitrate synthases (HCSs). Among the fungal lysine biosynthesis-related genes, only the HCS gene was duplicated in the course of evolution. It was recently reported that HCS of Saccharomyces cerevisiae has an additional function in nuclear activities involving chromatin regulation related to DNA damage repair, which is not related to lysine biosynthesis. Thus, it is possible that the bifunctionality is associated with HCS gene duplication. Phylogenetic analysis showed that duplication has occurred multiple times during evolution of the ascomycetous yeasts. It is likely that the HCS gene duplication in S. cerevisiae occurred in the course of Saccharomyces evolution. Although the nucleosome position profiles of the two $S$. cerevisiae HCS genes were similar in the coding regions, they were different in the promoter regions, suggesting that they are subject to different regulatory controls. S. cerevisiae has maintained HCS activity for lysine biosynthesis and has obtained bifunctionality.

\section{Introduction}

Organisms synthesize lysine from 2-oxoglutarate through $\alpha$ aminoadipate or from aspartic acid through diaminopimelate [1]. Animals cannot synthesize lysine. Fungi synthesize lysine through $\alpha$-aminoadipate [2-4]. The other eukaryotes synthesize lysine through diaminopimelate. Archaea and bacteria were also believed to synthesize lysine through diaminopimelate until it was reported that the extremely thermophilic bacterium Thermus thermophilus synthesizes lysine through $\alpha$-aminoadipate [5-8].

During lysine biosynthesis in the budding yeast Saccharomyces cerevisiae, $\alpha$-aminoadipate is synthesized from 2-oxoglutarate and acetyl-CoA by the enzymes Lys 20 or Lys 21 (homocitrate synthase [HCS]), Lys4 (homoaconitase), Lys12 (homoisocitrate dehydrogenase), and $\alpha$-aminoadipate aminotransferase [9]. Lysine is synthesized from $\alpha$-aminoadipate by the enzymes Lys2 (aminoadipate reductase), Lys5 (phosphopantetheinyl transferase which posttranslationally modifies Lys2), Lys9 (saccharopine dehydrogenase, glutamate forming), and Lys1 (saccharopine dehydrogenase, lysine forming) $[1,4]$.
It has been unclear why S. cerevisiae has 2 HCSs (Lys20 and Lys21). For example, homocitrate is mainly synthesized through Lys 21 during growth on ethanol, while under fermentative metabolism, Lys 20 and Lys 21 play redundant roles [11]. It was recently reported that Lys 20 of S. cerevisiae functions in nuclear activities involving chromatin regulation that are distinct from its previously established role in lysine synthesis [12]. Lys20 of S. cerevisiae is linked to the DNA damage repair process via the histone acetyltransferase Esa1 and the H2A.Z histone variant [12]. Thus, it is possible that this bifunctionality is associated with HCS gene duplication.

\section{Materials and Methods}

2.1. Phylogenetic Analyses. I selected 71 HCSs (31 from Saccharomycotina species, 30 from Pezizomycotina species, 2 from Taphrinomycotina species, and 8 from Basidiomycota species) based on BLASTP results in the fungal genome database at NCBI (http://www.ncbi.nlm.nih.gov/projects/ genome/guide/fungi/). Multiple alignments were generated with CLUSTAL W. A maximum likelihood tree was reconstructed using MEGA version 5 [10]. The WAG model was 


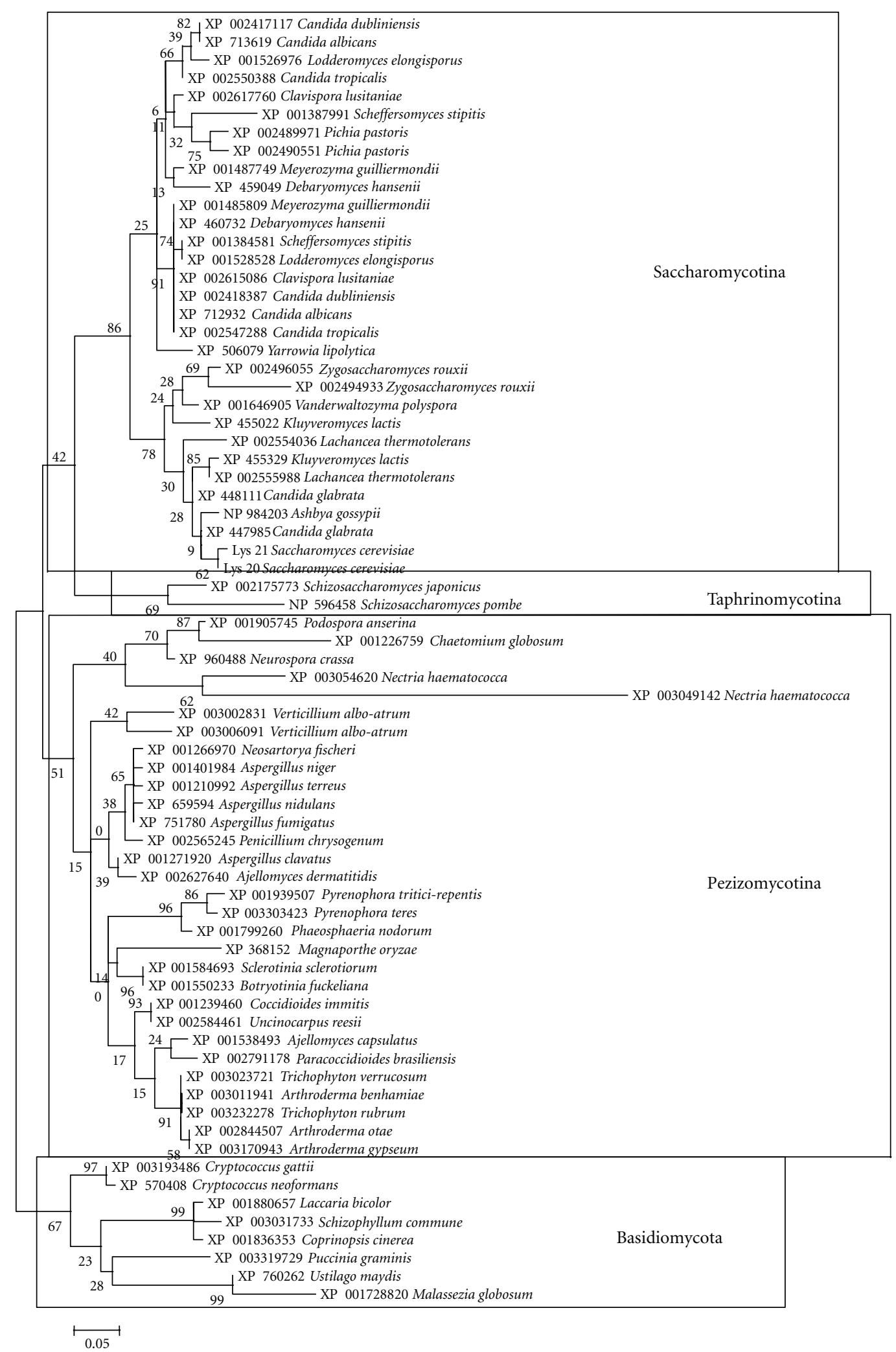

FIGURE 1: Phylogenetic relationships among 71 fungal homocitrate synthases. The phylogenetic tree was constructed based on multiple alignment with complete deletion of gap sites using the maximum likelihood method of MEGA software [10] with 100 bootstrap analyses. The WAG model was used as the amino acid substitution model. A total of 103 amino acid sites were considered. The $\gamma$-distributed rate was considered, and the number of discrete gamma categories was 3. The gamma was 0.81 ; the discrete rates were $0.14,0.65$, and 2.2 . 

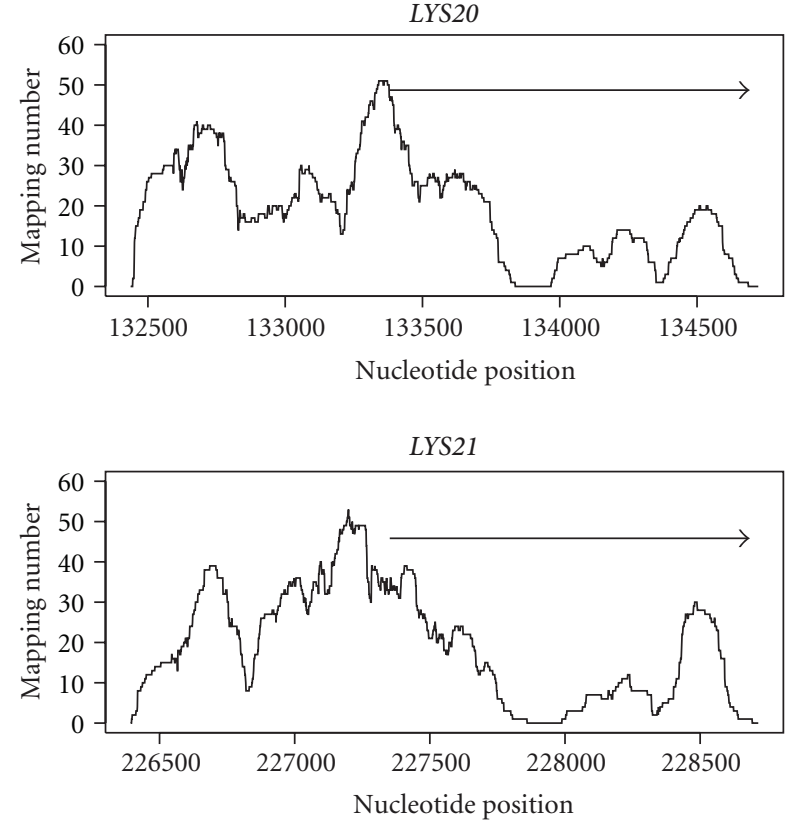

FIGURE 2: Mapping of nucleosomes around Saccharomyces cerevisiae LYS20 and LYS21. In this study, I used nucleosome position data from S. cerevisiae BY4741 [13]. Based on each nucleosomal DNA fragment sequence, nucleosomal mapping numbers were estimated for each nucleotide position [14]. Arrows indicate the coding region.

used as the amino acid substitution model. The nearest neighbor interchange was used as the maximum likelihood heuristic method. The $\gamma$-distributed rate was considered, and the number of discrete gamma categories was 3 .

2.2. Nucleosome Position Comparison. Nucleosome positioning was used to compare gene promoter regions. I used nucleosome position data from S. cerevisiae BY4741 [13]. The nucleosome position profiles were compared between the promoter (1000 bases upstream of the translational start site) and coding regions (between the translational start and end site) of the HCS genes, according to a previously described method [14]. Similarity between the two nucleosome position profiles was estimated using the Spearman's rank correlation coefficient.

\section{Results and Discussion}

The HCS phylogenetic tree (Figure 1) indicates that the HCS gene has been duplicated multiple times in the course of ascomycete evolution. The $31 \mathrm{HCS}$ s of the Saccharomycotina species (ascomycetous yeasts) are encoded in 17 organisms. In contrast, the $30 \mathrm{HCSs}$ of the Pezizomycotina species (filamentous ascomycetes) are encoded in 28 organisms. Thus, 14 of the 17 Saccharomycotina species and 2 of the 28 Pezizomycotina species have 2 HCSs (Figure 1).

Gene duplication is not found in LYS1, LYS2, LYS5, LYS9, and their homologues [15]. In addition, no duplication was found in LYS4, LYS12, and their homologues (data not shown). Therefore, among the fungal lysine biosynthesisrelated genes, only the HCS gene has been duplicated. Phylogenetic analysis of HCSs in ascomycetous yeasts showed that the S. cerevisiae HCSs (Lys20 and Lys21) are most closely related to each other (Figure 1), suggesting that HCS gene duplication occurred during evolution of the genus Saccharomyces. On the other hand, all Saccharomycotina species except Ashbya gossypii, Vanderwaltozyma polyspora, and Yarrowia lipolytica have duplicated HCS genes (Figure 1). Thus, HCS gene duplication may be related to genome duplication events in Saccharomycotina [16-18].

In addition to the phylogenetic analysis based on HCS amino acid sequences, I compared the nucleosome positioning of LYS20 and LYS21. Interestingly, nucleosomes were mapped to the HCS gene promoters more often than to the coding regions (Figure 2). Nucleosome position profiles in the coding regions were highly correlated (Spearman's rank correlation coefficient $=0.833$ ) between LYS20 and LYS21. On the other hand, those in the gene promoter regions were poorly correlated (Spearman's rank correlation coefficient $=0.396)$. This result suggests that these 2 HCS genes have different regulatory systems.

On the other hand, LYS20 expression is most similar to LYS21 expression, and LYS21 is most similar to LYS20 expression, based on the SPELL version 2.0.2 [19]. In addition, recent comparative analyses of orthologous genes in evolutionarily close yeasts indicated that divergence of nucleosome positioning is not correlated with divergence of gene expression [20, 21].

Although HCS (Lys20 and Lys21) is located in the nucleus of S. cerevisiae [22], HCS is located in the cytoplasm of Penicillium chrysogenum $[23,24]$. P. chrysogenum has a single HCS gene (Figure 1). The phylogenetic tree (Figure 1) showed that gene duplication is not found in Basidiomycota and Taphrinomycotina. In addition, gene duplication has occurred rarely in Pezizomycotina, suggesting that a common ancestor of the Dikarya lacked the nuclear function of chromatin regulation. Considering that duplication of the HCS gene occurred in a limited number of ascomycetes, it may not be an essential event in the evolution of Dikarya. I hypothesize that after divergence of the phyla Ascomycota and Basidiomycota, S. cerevisiae obtained HCS bifunctionality.

\section{Acknowledgment}

This work was supported in part by a research grant from the Institute for Fermentation, Osaka (IFO), Japan.

\section{References}

[1] H. Xu, B. Andi, J. Qian, A. H. West, and P. F. Cook, "The $\alpha-$ aminoadipate pathway for lysine biosynthesis in fungi," Cell Biochemistry and Biophysics, vol. 46, no. 1, pp. 43-64, 2006.

[2] H. P. Broquist, "Lysine biosynthesis (yeast)," Methods in Enzymology, vol. 17, no. 2, pp. 112-113, 1971.

[3] H. J. Vogel, "Distribution of lysine pathways among fungi: evolutionary implications," American Naturalist, vol. 98, pp. 435446, 1964. 
[4] T. M. Zabriskie and M. D. Jackson, "Lysine biosynthesis and metabolism in fungi," Natural Product Reports, vol. 17, no. 1, pp. 85-97, 2000.

[5] N. Kobashi, M. Nishiyama, and M. Tanokura, "Aspartate kinase-independent lysine synthesis in an extremely thermophilic bacterium, Thermus thermophilus lysine is synthesized via $\alpha$ aminoadipic acid not via diaminopimelic acid," Journal of Bacteriology, vol. 181, no. 6, pp. 1713-1718, 1999.

[6] T. Kosuge and T. Hoshino, "Lysine is synthesized through the $\alpha$-aminoadipate pathway in Thermus thermophilus," FEMS Microbiology Letters, vol. 169, no. 2, pp. 361-367, 1998.

[7] H. Nishida, M. Nishiyama, N. Kobashi, T. Kosuge, T. Hoshino, and H. Yamane, "A prokaryotic gene cluster involved in synthesis of lysine through the amino adipate pathway: a key to the evolution of amino acid biosynthesis," Genome Research, vol. 9, no. 12, pp. 1175-1183, 1999.

[8] H. Nishida, "Distribution of genes for lysine biosynthesis through the aminoadipate pathway among prokaryotic genomes," Bioinformatics, vol. 17, no. 2, pp. 189-191, 2001.

[9] I. Iraqui, S. Vissers, M. Cartiaux, and A. Urrestarazu, "Characterisation of Saccharomyces cerevisiae ARO8 and ARO9 genes encoding aromatic aminotransferases I and II reveals a new aminotransferase subfamily," Molecular and General Genetics, vol. 257, no. 2, pp. 238-248, 1998.

[10] K. Tamura, D. Peterson, N. Peterson, G. Stecher, M. Nei, and S. Kumar, "MEGA5: molecular evolutionary genetics analysis using maximum likelihood, evolutionary distance, and maximum parsimony methods," Molecular Biology and Evolution, vol. 28, no. 10, pp. 2731-2739, 2011.

[11] H. Quezada, C. Aranda, A. DeLuna et al., "Specialization of the paralogue LYS21 determines lysine biosynthesis under respiratory metabolism in Saccharomyces cerevisiae," Microbiology, vol. 154, no. 6, pp. 1656-1667, 2008.

[12] E. M. Scott and L. Pillus, "Homocitrate synthase connects amino acid metabolism to chromatin functions through Esa1 and DNA damage," Genes and Development, vol. 24, no. 17, pp. 1903-1913, 2010.

[13] T. Matsumoto, C.-S. Yun, H. Yoshikawa, and H. Nishida, "Comparative studies of genome-wide maps of nucleosomes between deletion mutants of elp3 and hos 2 genes of Saccharomyces cerevisiae," Plos ONE, vol. 6, no. 1, Article ID e16372, 2011.

[14] H. Nishida, "Calculation of the ratio of the mononucleosome mapping number to the dinucleosome mapping number for each nucleotide position in the Aspergillus fumigatus genome," Open Access Bioinformatics, vol. 1, pp. 1-6, 2009.

[15] H. Nishida and M. Nishiyama, "What is characteristic of fungal lysine synthesis through the $\alpha$-aminoadipate pathway?" Journal of Molecular Evolution, vol. 51, no. 3, pp. 299-302, 2000.

[16] M. Kellis, B. W. Birren, and E. S. Lander, "Proof and evolutionary analysis of ancient genome duplication in the yeast Saccharomyces cerevisiae," Nature, vol. 428, no. 6983, pp. 617624, 2004.

[17] R. T. Morris and G. Drouin, "Ectopic gene conversions in the genome of ten hemiasomycete yeast species," International Journal of Evolutionary Biology, vol. 2011, Article ID 970768, 11 pages, 2011.

[18] K. H. Wolfe and D. C. Shields, "Molecular evidence for an ancient duplication of the entire yeast genome," Nature, vol. 387, no. 6634, pp. 708-713, 1997.

[19] M. A. Hibbs, D. C. Hess, C. L. Myers, C. Huttenhower, K. Li, and O. G. Troyanskaya, "Exploring the functional landscape of gene expression: directed search of large microarray compendia," Bioinformatics, vol. 23, no. 20, pp. 2692-2699, 2007.

[20] I. Tirosh, N. Sigal, and N. Barkai, "Divergence of nucleosome positioning between two closely related yeast species: genetic basis and functional consequences," Molecular Systems Biology, vol. 6 , article $365,2010$.

[21] K. Tsui, S. Dubuis, M. Gebbia et al., "Evolution of nucleosome occupancy: conservation of global properties and divergence of gene-specific patterns," Molecular and Cellular Biology, vol. 31, no. 21, pp. 4348-4355, 2011.

[22] S. Chen, J. S. Brockenbrough, J. E. Dove, and J. P. Aris, "Homocitrate synthase is located in the nucleus in the yeast Saccharomyces cerevisiae," Journal of Biological Chemistry, vol. 272, no. 16, pp. 10839-10846, 1997.

[23] O. Bañuelos, J. Casqueiro, S. Steidl, S. Gutiérrez, A. Brakhage, and J. F. Martín, "Subcellular localization of the homocitrate synthase in Penicillium chrysogenum," Molecular Genetics and Genomics, vol. 266, no. 5, pp. 711-719, 2001.

[24] W. M. Jaklitsch and C. P. Kubicek, "Homocitrate synthase from Penicillium chrysogenum. Localization, purification of the cytosolic isoenzyme, and sensitivity to lysine," Biochemical Journal, vol. 269, no. 1, pp. 247-253, 1990. 

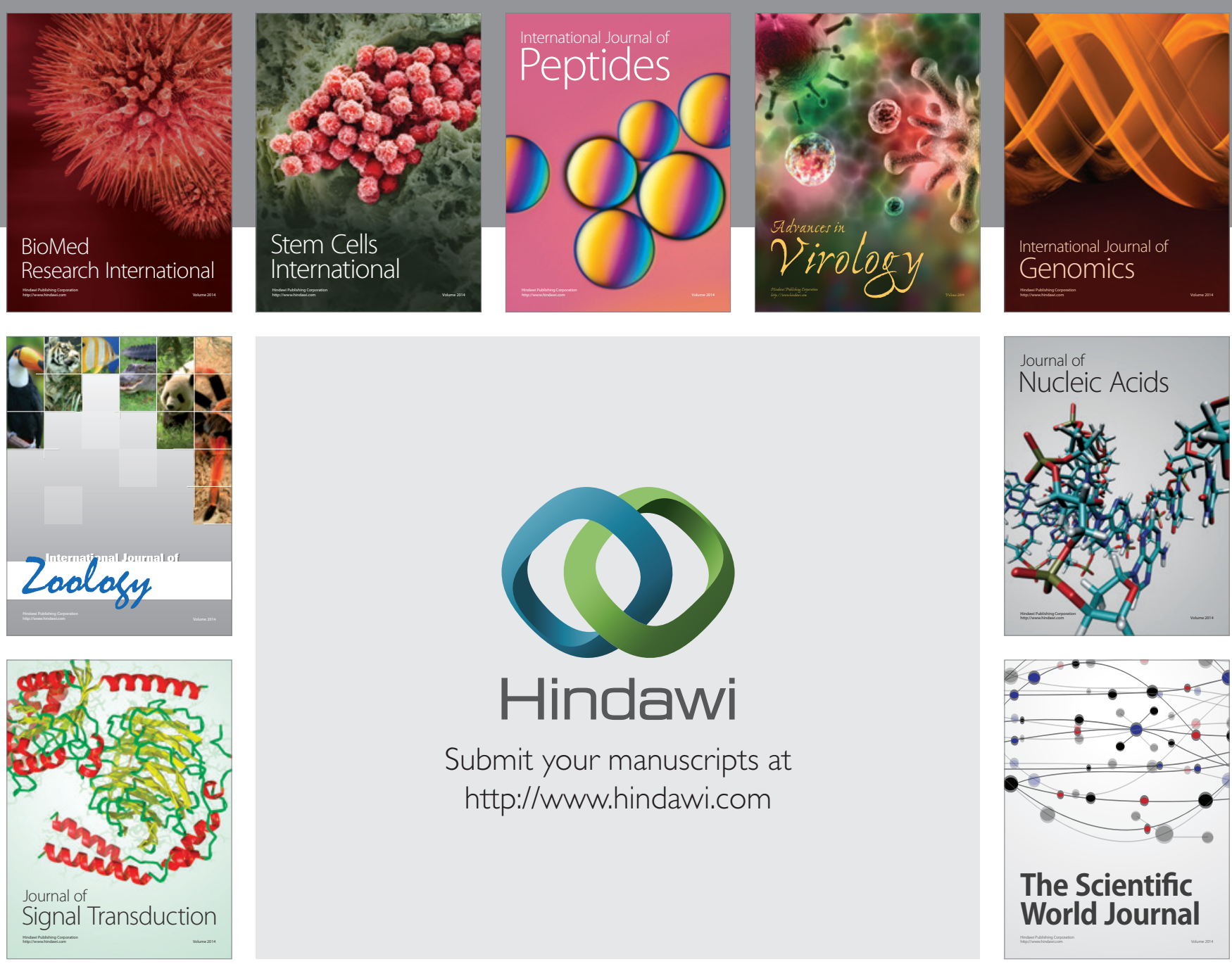

Submit your manuscripts at

http://www.hindawi.com
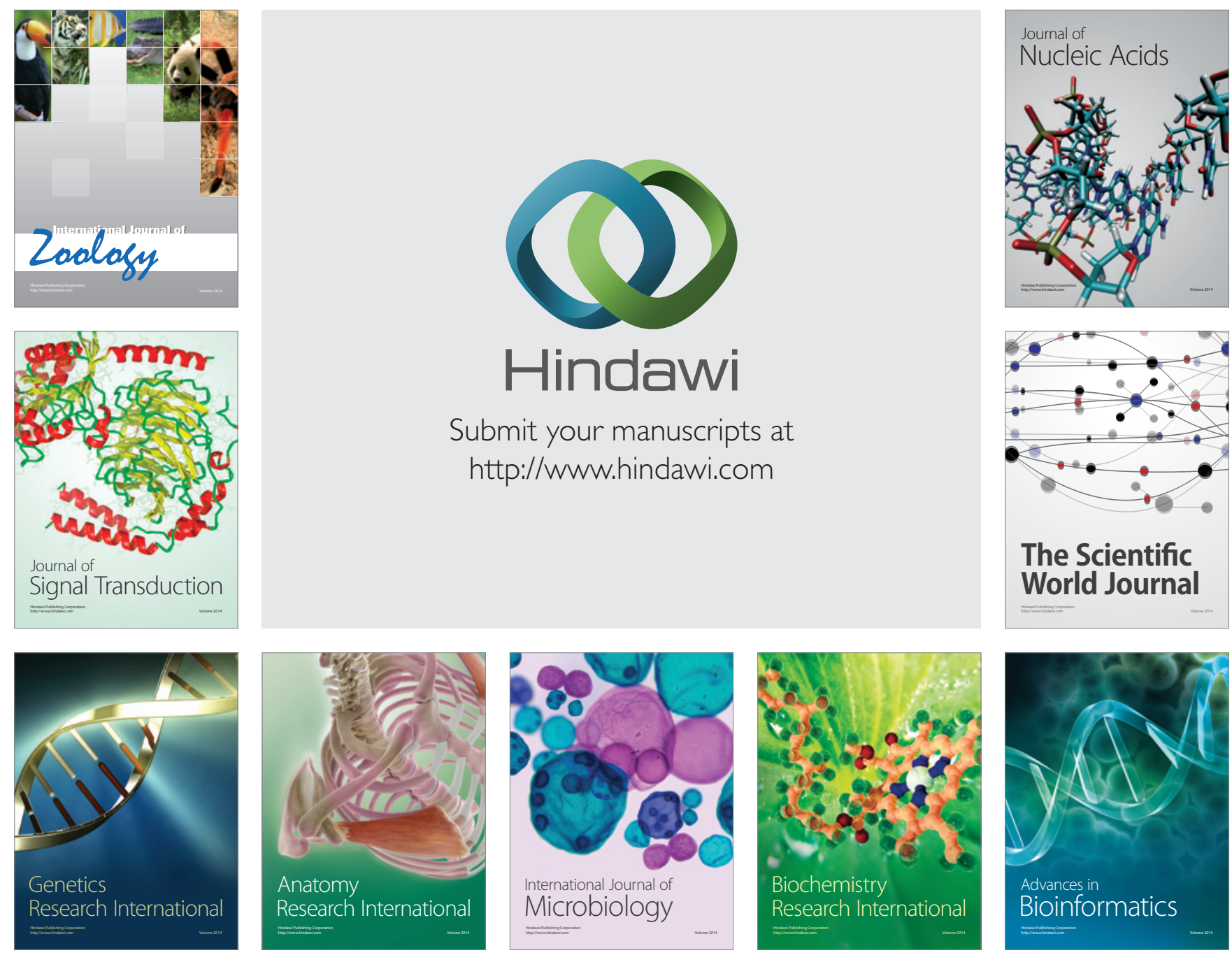

The Scientific World Journal
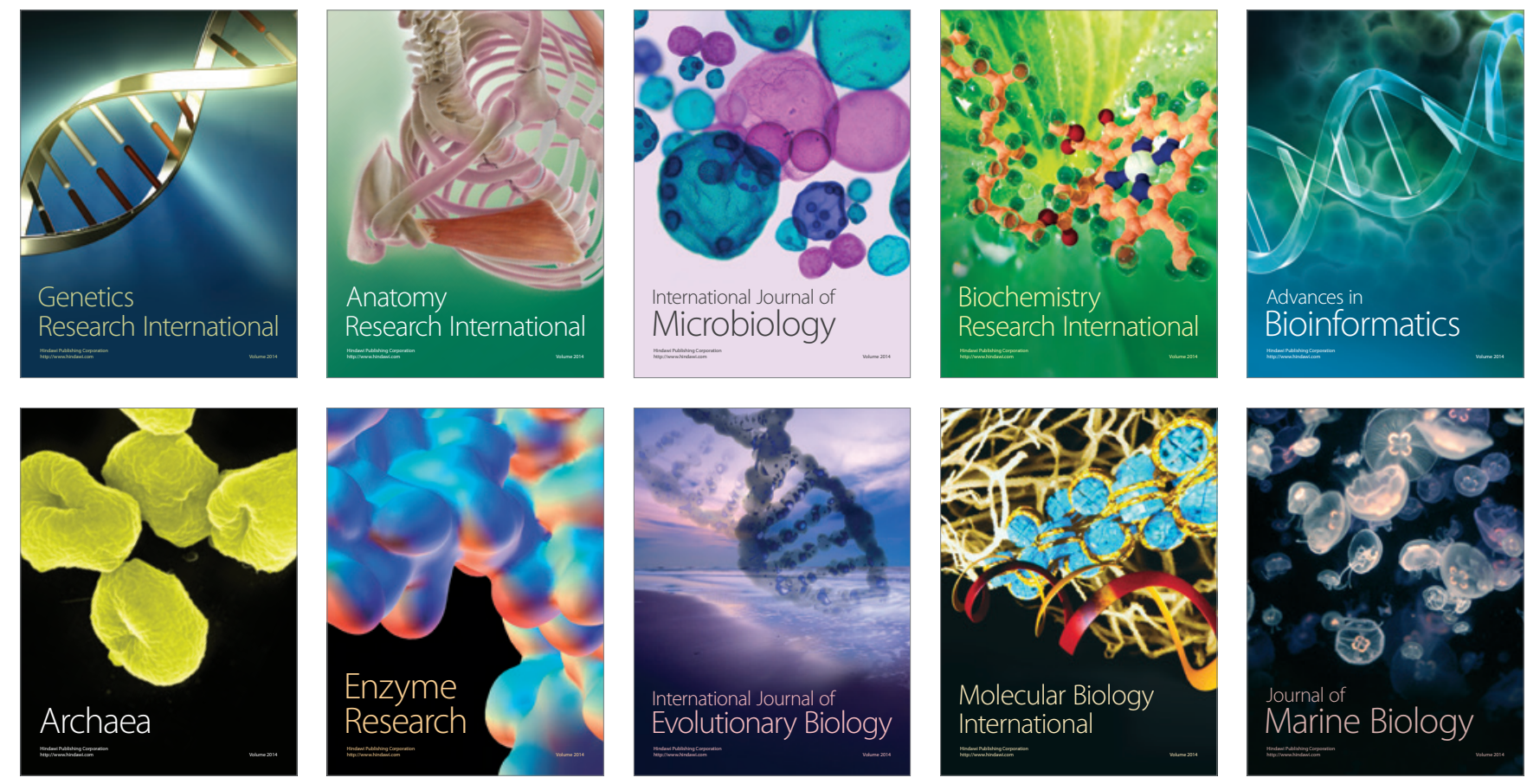